\title{
Reprocessable thermoset soft actuators
}

\author{
Yang Yang, ${ }^{[a]}$ Eugene M. Terentjev, ${ }^{[b]}$ Yubai Zhang, ${ }^{[a]}$ Qiaomei Chen, ${ }^{[a]}$ Yuan Zhao, ${ }^{[a]}$ Yen Wei, ${ }^{[a]}$ and \\ Yan $\mathrm{Ji}^{*[a]}$
}

\begin{abstract}
To construct 3D soft actuators, the widely-used traditional thermosets are good candidate due to their excellent stability, while it is generally acknowledged that they cannot be reprocessed. Here we show that the time-temperature equivalence principle enables the reprocessing of traditional liquid crystalline epoxy thermosets (LCETs) into 3D soft actuators. Even though the transesterification reaction of LCETs is too slow to be ignored, it is enough to induce a topology rearrangement and subsequent reprocessing when prolonging the transesterification time according to above principle. So LCETs can be aligned by a simple procedure. The alignment is quite stable at high temperature and remains after above 1,000 heating-cooling actuation cycles. The resulted 3D soft actuators are also remouldable, reprogrammable, reconfigurable, weldable, selfhealable, recyclable and stable, which is impossible for any previous traditional thermosets but very appealing for their applications.
\end{abstract}

\section{Introduction}

The rapid expansion of work in soft robotics, wearable devices, artificial muscles, cell scaffolds, micromanipulators and so on leads to a soaring demand on soft actuators made of deformable materials to transform external energy (light, electricity, heat, etc.) directly into mechanical deformation. ${ }^{[1]}$ Due to the high dimensional stability and good mechanical properties, thermosets are widely chosen for the construction of soft actuators. Thermosets are permanently crosslinked polymers that are irreversibly hardened by curing from a soft solid or viscous liquid prepolymer. ${ }^{[2]}$ Curing, resulting in chemical reactions, creates extensive cross-linking between polymer chains to produce an infusible and insoluble polymer network, which is useful for stability, but causes much inconvenience in the post-processing of soft actuators, and also limits the geometry of the actuators to relatively simple shapes while complex geometry plays an important role to define the function. Moreover, once the soft actuator made of traditional thermoset is formed, its 3D shape cannot be changed. In many situations, small modification can make a damaged or abandoned actuator useful for another purpose, which is helpful in reducing waste and taking full advantage of resources towards a sustainable society. Recently, covalently crosslinked polymers with dynamic

\footnotetext{
[a] Dr. Y. Yang, Y. Zhang, Dr. Q. Chen, Dr. Y. Zhao, Prof. Y. Wei, Prof. Y. Ji

The Key Laboratory of Bioorganic Phosphorus Chemistry \&

Chemical Biology (Ministry of Education), Department of Chemistry,

Tsinghua University, Beijing 100084, China

E-mail: jiyan@mail.tsinghua.edu.cn

[b] Prof. E. M. Terentjev

Cavendish Laboratory, University of Cambridge, Cambridge CB3 OHE, UK
}

Supporting information for this article is given via a link at the end of the document. covalent bonds, such as liquid crystalline vitrimers (xLCEs) and/or covalently exchangeable networks, provided an extraordinary solution to change the actuators' shapes after they have been fabricated ${ }^{[3]}$. Moreover, the exchangeable links enable these polymers to be remouldable, weldable, selfhealable, and fully recyclable ${ }^{[3-4]}$. However, they have different shortcomings. For example, xLCEs have a lack of stability. ${ }^{[3 e, 34,5]}$ Covalently crosslinked polymers with photo-chemical reactions, such as thiol-acrylate reaction, are based on plasticity instead of reprocessability. ${ }^{[5 h]}$ Both the construction of complex 3D geometries and the change of fixed shapes after actuator fabrication require the reprocessing of actuator materials.

So far, for traditional thermosets, reprocessing remained impossible. Vitrimers were firstly obtained by adding a transesterification catalyst into classcial epoxy thermosets cured by diacids ${ }^{[4 \mathrm{~d}, 4 \mathrm{e}]}$. The compositional difference between epoxy vitrimers and classical epoxies is only that there is a notable amount of transesterification catalyst in the epoxy vitrimers, while there is no such catalsyt in the classical epoxy networks. This small difference in composition leads to a huge processing difference: epoxy vitrimers can be easily reprocessed while classical epoxy thermostes cannot. For vitrimers, there is a special temperature defined as topology freezing transition temperature $\left(T_{v}\right)^{[6]}$. Below $T_{v}$, the network topology is frozen, while above $T_{v}$ the network can be changed. This temperature can be easily detected by dilatometry test; while for the classical epoxy thermosets without a catalyst this temperature cannot be identified. This was always taken as an indication that the topology of the classical epoxy cannot be changed. From the stress-relaxation tests, the relaxation of classical epoxy thermoset is exteremmly slow (Figure S6), which in the past was regarded as a proof that classical epoxy cannot be reprocessed.

Contrary to the traditional perception that traditonal epoxy thermosets cannot be reprocessed, here we present an example of classical liquid crystalline epoxy thermosets (LCETs), without a catalyst to promote transesterification, to be reprocessed into chosen shapes of soft actuators. Since the work of Leiblet et al. It was understood that the catalyst is required to allow plastic flow above the vitrification temperature. However, we show that transesterification still occurs in LCET, although at a much lower rate, and therefore the same reprocessing is possible as in vitrimers. Transesterification is an equilibrium and reversible reaction, and the presence of catalyst only lowers its activation energy, making the bond-exchange reactiuon faster. So a longtime transesterification is able to change the topology of the network, and further, reprocess LCETs. The resulting LCET actuators combine the stable actuation of tradiational themosets with the unique reprocessability of xLCE vitrimers. Moreover this brings some advantages that xLCEs do not have. For example, the 3D soft actuators made of aligned LCETs can be reprogrammed stepwise and locally by heat, which is impossible to be achieved by previous xLCE due to the fast transesterification inducing an immediate destruction of the 
alignment. Moreover, it provides a feasible method to use heat to weld/assemble aligned LCETs into complicated structures without destroying the alignment, which could not be realized in the past either. Both reprogramming and welding LCET actuators will make it much more convenient to build complex 3D actuators with reversible and stable actuations. When combined with a light-responsive component, the LCET composites can also be reprocessed to $3 \mathrm{D}$ soft actuators driven by light. In other words, even though LCETs are traditional thermosets, instead of typical vitrimers, they can be reprocessed into 3D soft actuators.

The traditional thermoset actuator materials we used here belong to classical liquid crystalline epoxy thermosets ${ }^{[7]}$. This is a

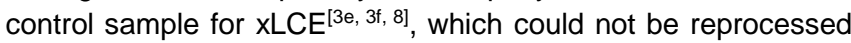
after cured. The thermoset nature prevented the alignment after curing to form the monodomain liquid crystalline elastomer (LCE), which is necessary for spontaneous large-scale reversible shape changes between isotropic phase and liquidcrystal phase in response to external stimuli. ${ }^{[3, \text { 9] }}$ Moreover, it is widely believed that their transesterification reaction was much too slow to induce a topology change ${ }^{[10]}$, which could be seen from experimental results. For example, from the dilatometry test of LCETs we did before, there was no obvious signal indicating a transesterification-induced topology change yet. ${ }^{[3]]}$ And from the stress-relaxation test (inset in Figure S8), we can see that compared to XLCE, the relaxation of LCET is so slow that we almost thought it could not relax. ${ }^{[3]]}$

Our strategy to reprocess the traditional LCETs originated from our recent serendipitous finding ${ }^{[6 b]}$. In our recent work to measure $T_{v}$ by aggregation induced emission (AIE)-probes technique, we found that like vitrimer, which contains a certain amount of transesterification catalyst, the traditional epoxy thermoset without any catalysts also has a same topology freezing transition, which indicates a dramatic change of reaction rate. ${ }^{[6 \mathrm{~b}]}$ The existence of this transition, with or without the catalyst, indicates that the traditional epoxy thermosets should share a similar topology rearrangement potential and consequent reprocessability as typical vitrimers. As the transesterification reaction of epoxy thermosets without a catalyst is too slow to change the network topology in ordinary circumstances, what should we do to make these LCETs reprocessable? To accelerate the transesterification reaction, increasing the temperature is a valid method, since this is an activation process. But the temperature used to reprocess $\mathrm{XLCE}$ is already high enough, and the materials will be decomposed if we increase temperature even further. Another effective approach is to add a catalyst. But by doing so LCETs would become classical vitrimers, the xLCE we studied earlier that lacks long-term thermal stability. This led us to think of the timetemperature equivalence principle ${ }^{[11]}$. According to this principle, the same mechanical responses (such as stress-relaxation) can be observed for a long enough time at a lower temperature, or for a short time at higher temperatures. ${ }^{[11 b]}$ That is, prolonging the observation time has the same effect as increasing the temperature. So we suppose that the stress-relaxation and topology rearrangement can still be achieved by prolonging the transesterification time. We verified this hypothesis using a common epoxy thermoset (not mesogenic LCET) prepared by reacting diglycidyl ether of bisphenol $A$ with adipic acid (Figure
S1). The stress-relaxation curves (Figure S6a) clearly show that the stress of this common epoxy (without catalyst) does relax at about $120^{\circ} \mathrm{C}$, only much slower than that of vitrimers with different catalyst loadings due to the thermally-activated transesterification. Moreover, like vitrimer, it relaxes at different rates at different temperatures (Figure S6b). The long-time transesterification-induced network rearrangement is sufficient to fix the new shape (Figure S6c), and even more, recycle the fragments by hot-pressing (Figure $S 6 \mathrm{~d}$ ) in $2 \mathrm{~h}$ at $180^{\circ} \mathrm{C}$. Further, from Figure $S 8$, the traditional LCETs could also relax the stress at $180^{\circ} \mathrm{C}$ for a long time, so they should also be reprocessable so as to make monodomain LCE actuators and form 3D shapes.

\section{Results and Discussion}

As a demonstration, here we use one traditional LCET prepared by reacting diglycidyl ether of 4, 4'-dihydroxybiphenyl with sebacic acid at $180^{\circ} \mathrm{C}$ for $6 \mathrm{~h}$ (Figure 1a). The resulting network was insoluble at $180^{\circ} \mathrm{C}$ when swelled in trichlorobenzene (Figure S3), indicating a fully crosslinked polymer. The initially obtained LCET is unaligned (see details in Supporting information). The moulding test (Figure 1b) clearly proves that a slow transesterification can change the topology of the network, so it is possible to align the mesogens and reconfigure the orientation by transesterification bond exchange over a long time. The detailed aligning procedure is shown in Figure 1c (i): firstly, an unaligned polydomain LCET strip is stretched by $30 \%$ at $180^{\circ} \mathrm{C}$ (the elongation at break at $180^{\circ} \mathrm{C}$ is $\sim 45 \%$, Figure S9); secondly, the stretched strip is kept at $180^{\circ} \mathrm{C}$ for $2 \mathrm{~h}$, which is much longer than in the previous methods ${ }^{[3 \mathrm{f}, 5 \mathrm{f}]}$; during this time a uniaxial alignment is programmed into the network. We could also use a lower temperature (e.g. $\left.100^{\circ} \mathrm{C}\right)$ for a much longer time for the sample alignment in liquid-crystal phase. Here we choose $180^{\circ} \mathrm{C}$ to shorten the processing time, which results in its isotropic-genesis alignment ${ }^{[12]}$. The aligned LCET has a spontaneous reversible elongation between liquidcrystal phase and isotropic phase of $64 \%$ (Figure 1d), which is much larger than the pre-strain of $30 \%$. This is because both the pre-stretching and the 2-hour transesterification were done in the isotropic phase, and their role is merely to provide a uniform guiding direction for the LC order to follow on cooling. When such a 'weakly anisotropic' LCET goes through an isotropic-LC phase transition, its mesogens will align along the prescribed uniform direction, and the shape elongate further. The X-ray diffraction (XRD) image (Figure 1e) and polarizing optical microscope (POM) pictures (Figure S10) clearly indicate a good alignment.

Similar to xLCEs, LCETs here allow robust alignment and flexible reconfiguration for various soft actuators. For example, an aligned LCET with reversible bending movement (Figure 1f) is obtained by binding a stretched film to an iron-mould and keeping it at $180^{\circ} \mathrm{C}$ for $2 \mathrm{~h}$. By twining a stretched LCET strip round a wire (Figure 1g), a spiral actuator is obtained. For another example, we produced a dome-shape 3D actuator (Figure $1 \mathrm{~h}$ and video 1 ), which becomes flat as temperature goes up and reappears on cooling. The dome-shape sample has 
an initial height of about $0.9 \mathrm{~mm}$. It was remoulded by placing an unaligned LCET film on the top of the pin of a punch, covering with the die mould, gently pressing die mould for pin to insert into its hole, keeping it at $180^{\circ} \mathrm{C}$ for $2 \mathrm{~h}$, and at last taking off the die mould and peeling it out. The wall and roof of dome-shape are aligned while other parts remain unaligned. This configuration is very important for the use of tactile display technology, such as Braille displays.

Unlike xLCEs, the aligned LCETs here have a much more stable alignment, superior to all the reported xLCEs. The alignment of the originally reported XLCE disappears immediately (within 5 seconds) at high temperature (above the isotropic-liquid crystal phase transition temperature $\left(T_{\mathrm{i}}\right)$ range) or after several reversible actuation cycles due to catalyst accelerated transesterification. $\left.{ }^{33}, 3 \mathrm{f}, 5 \mathrm{~g}\right]$ Even though the alignment stability of the xLCE could be improved by decreasing the transesterification catalyst content, it would also disappear in 90 minutes at $180^{\circ} \mathrm{C} .{ }^{[5]}$ But here, the alignment of LCET remains excellent even kept it at $180^{\circ} \mathrm{C}$ for $12 \mathrm{~h}$. It has little fatigue after repeating the reversible elongation-contraction actuation between isotropic phase and liquid-crystal phase for 1000 times. We measured the first 70 heating-cooling cycles and another 5 cycles after repeating 1000 cycles by dynamic mechanical analyser (DMA). From Figure 1j, we can see the alignment remains good.

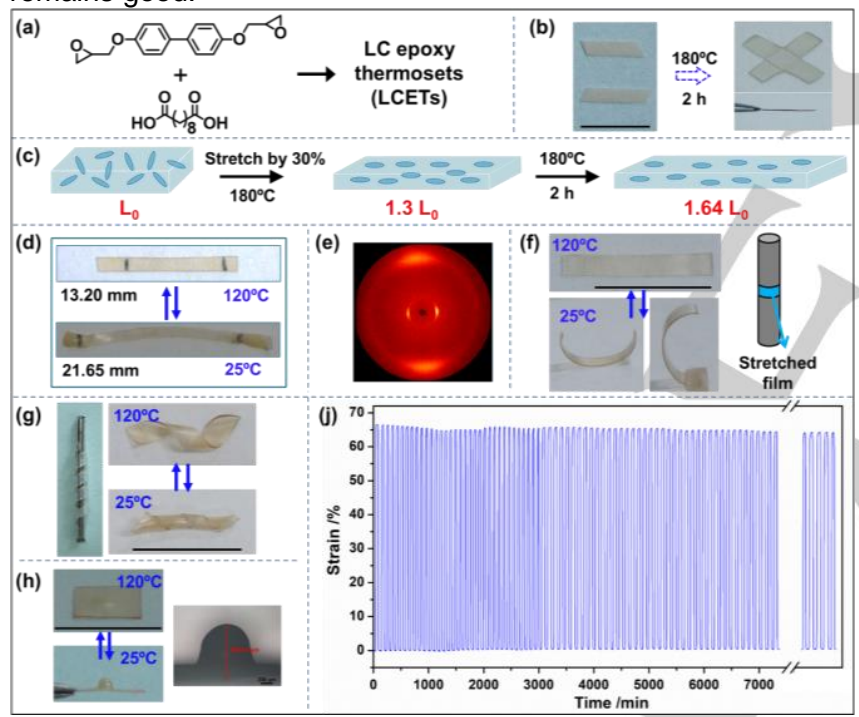

Figure 1. (a) The synthesis of unaligned LCETs. (b) Two unaligned LCET films were compress-moulded together at $180^{\circ} \mathrm{C}$ for $2 \mathrm{~h}$ as a result of the transesterification. From the side view of the joint sample, the thickness of the overlap area is the thickness of one film. Scale bar: $1 \mathrm{~cm}$. (c) The aligning procedure of LCET. (d) Reversible thermal stretching-contraction actuation of an aligned LCET between $120^{\circ} \mathrm{C}$ (isotropic phase) and $25^{\circ} \mathrm{C}$ (liquid-crystal phase). The extension ratio is $64 \%$. (e) XRD image of the aligned LCET, which shows a well-aligned order. (f) Reversible bending-unbending actuation of an aligned LCET film. Scale bar: $1 \mathrm{~cm}$. $(\mathrm{g})$ Reversible actuation of an aligned spiral LCET. Scale bar: $1 \mathrm{~cm}$. (h) An aligned dome-shape LCET actuator. Scale bar: $1 \mathrm{~cm}$. (j) The contraction-elongation actuation of the aligned LCET for the first 70 heating-cooling cycles and another 5 cycles after repeating 1000 cycles between $150^{\circ} \mathrm{C}$ (in isotropic phase) and $80^{\circ} \mathrm{C}$ (in liquid-crystal phase) measured by DMA.
This stable alignment allows aligned LCETs to be reprogrammed stepwise or locally into 3D soft actuators by heat, which has not be demonstrated in neither traditional thermosets nor xLCEs. Previously, it is hard to reprogram the aligned xLCE actuators step by step by heat because the actuators would totally lose the existing alignments due to the fast transesterification. Moreover, when reprogramming xLCEs, the extra tension would induce a lower activation energy and a lower $\mathrm{T}_{\mathrm{v}}$, which leads to an easier destruction to the alignments. But reprogramming them stepwise is necessary in many cases, for example when just a tiny shape change is needed or when the new shape needs to be built based on the old one, also, it is energy-saving and sustainable. LCET actuators here can be stepwise reprogrammed due to the stable actuation, as the alignment of LCETs remains good after two-hour transesterification, in spite of the external force. For example, by dividing a circle into twelve sectors (Figure $2 \mathrm{a}$, i) and locally aligning each interval sector to bending actuation, the sample opens in liquid-crystal phase and closes in isotropic phase reversibly (Figure $2 \mathrm{a}$, ii). It can be further reprogrammed to an iris-like actuator by aligning the remaining sectors to bending actuation without destroying the pre-existing alignments (Figure $2 \mathrm{a}$, iii). If light-responsive components, such as nanoparticles with photo-thermal effects (e.g., carbon nanotubes and graphenes), are introduced, the opening-closing cycle of iris-like LCE actuator is possible to be actuated by sunlight, which shows great potentials in biomimetic materials for camera aperture and aerospace materials. But previously reported irislike LCEs were prepared by using either custom-designed magnetic fields with embedded stretchable heaters ${ }^{[13]}$ or a liquidcrystal cell and a polarization converter for orientations ${ }^{[14]}$, whose procedures and operations were complicated. And their actuations could only be triggered by either an electricalactuated-thermal means ${ }^{[13]}$ or UV light ${ }^{[14]}$, and could not respond to sunlight. Additionally, this aligning method enables LCETs to be locally reconfigured into $3 \mathrm{D}$ soft actuators by heat. For example, when binding a stretched four-petal LCET (two diagonal petals were stretched by $15 \%$, and the other diagonal petals were stretched by $30 \%$ ) to a metal ball (Figure $2 \mathrm{~b}$ ii) and keeping it at $180^{\circ} \mathrm{C}$ for $2 \mathrm{~h}$, a flower-like actuator (Figure $2 \mathrm{~b} \mathrm{i}$ ) is produced. The actuation degrees of petals are different in one film.

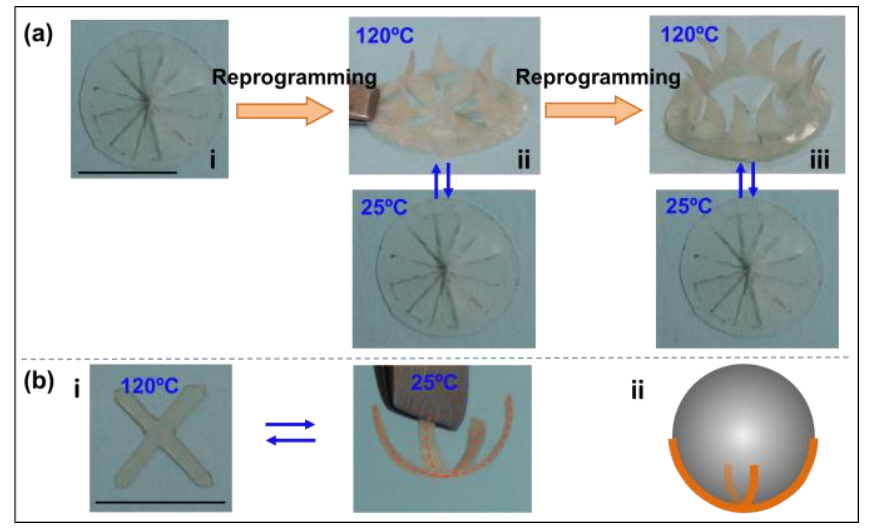


Figure 2. (a) Reprogramming LCET stepwise to a 3D iris-like actuator with reversible actuations. Scale bar: $1 \mathrm{~cm}$. (b) A reconfigured flower-like actuator. Scale bar: $1 \mathrm{~cm}$.

This stable alignment also allows aligned LCETs to be welded/assembled into one system to produce complex soft actuators by heat. In the past, aligned xLCEs could not be welded by heat (as the alignment would be lost), but could only be welded by light when light-responsive component was introduced (as light could be controlled locally). But here, it is much more convenient to use heat to construct complex 3D shapes by welding. Only the alignment of the joint part is damaged to some extent and the rest of alignment remains good, as only the joint part was welded under external force. For example, we made a triangle actuator (Figure $3 \mathrm{a}$ ) and a square actuator (Figure $3 \mathrm{~b}$ ) respectively by welding aligned strips end to end. The overlaps of the triangle and the square are bonded together by covalent bonds, which avoids the delamination between strips. For another example, we could make robust tubular actuator (Figure 3c) and subuliform actuator (Figure 3d) with reversible expanding-shrinking actuation by aligning the LCET sheet, rolling up and welding the overlap. Tubular actuators show great potentials in soft robotics, as artificial blood vessels and so on. ${ }^{[3 h, 15]}$ Welding aligned LCET strips together provides a simple method to build netty actuators, whose pores enlarge in liquid-crystal phase and shrink in isotropic phase (Figure $3 e$ and $3 f$ ). The existing polymeric netty-structures are made by binding strips together to multi-layer, which tend to delaminate after repeated use. ${ }^{[16]}$

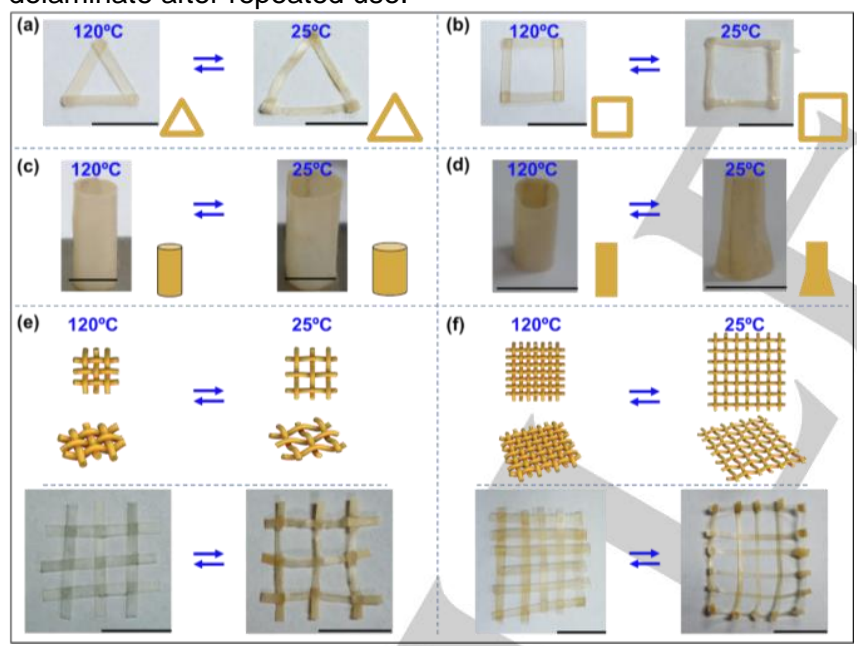

Figure 3. (a) Reversible actuation of a welded triangular LCET actuator. Scale bar: $1 \mathrm{~cm}$. (b) Reversible actuation of a welded square LCET actuator. Scale bar: $1 \mathrm{~cm}$. (c) Reversible expanding-shrinking of a tubular actuator. Scale bar: $1 \mathrm{~cm}$. (d) Reversible expanding-shrinking of a subuliform actuator. Scale bar: 1 $\mathrm{cm}$. (e) Reversible actuation of a welded netty actuator. Scale bar: $1 \mathrm{~cm}$. (f) Reversible actuation of another welded netty actuator. Scale bar: $1 \mathrm{~cm}$.

Besides pure LCETs, LCET-composites can also be reprocessed to produce 3D soft actuators by this method. They provide a feasible approach to reprocess LCETs by other stimuli, such as light. For example, carbon nanotubes (CNTs) doped above composites can be aligned by light owing to the strong photo-thermal effect of CNTs. CNTs absorb light energy and transfer it into thermal energy, ${ }^{[17]}$ triggering transesterification ${ }^{[3}$, ${ }^{18]}$ and consequently reconfiguring the mesogen orientation. By irradiating a stretched composite strip with the infrared light (808 $\mathrm{nm}$, intensity: $0.84 \mathrm{~W} / \mathrm{cm}^{2}$ ) for more than 30 minutes, the strip is aligned and the elongation is about $54 \%$ (Figure $4 \mathrm{a}$ ). Moreover, infrared light can be used to trigger its reversible actuation (intensity: $0.47 \mathrm{~W} / \mathrm{cm}^{2}$, the sample reaches $120^{\circ} \mathrm{C}$ in 10 seconds, Figure S11), which enables the composite to do mechanical work, for example, lifting a weight (Figure 4a ii and video 2). Light can also trigger reversible bending and spiral actuations (Figure 4b, 4c and video 3 ) as well. As light could be controlled remotely, locally and in-situ, light-controlled actuator shows great practical applications. For example, it can be used to control a circuit as the light is turned on and off (light intensity: $0.47 \mathrm{~W} / \mathrm{cm}^{2}$ ). As shown in Figure $4 \mathrm{~d}$ and video 4, when irradiating, the strip contracts and pulls the copper sheet up, so that the circuit is connected and the bulb shines. When turning the light off, the strip stretches and the copper sheet drops down, so the circuit is disconnected and the bulb goes out. Light can also trigger self-healing of the aligned composites. A needle pierced hole (Figure $4 \mathrm{e}$ i) and a cut (ii) are well healed by irradiating infrared light $\left(0.84 \mathrm{~W} / \mathrm{cm}^{2}\right)$ for $30 \mathrm{~min}$.

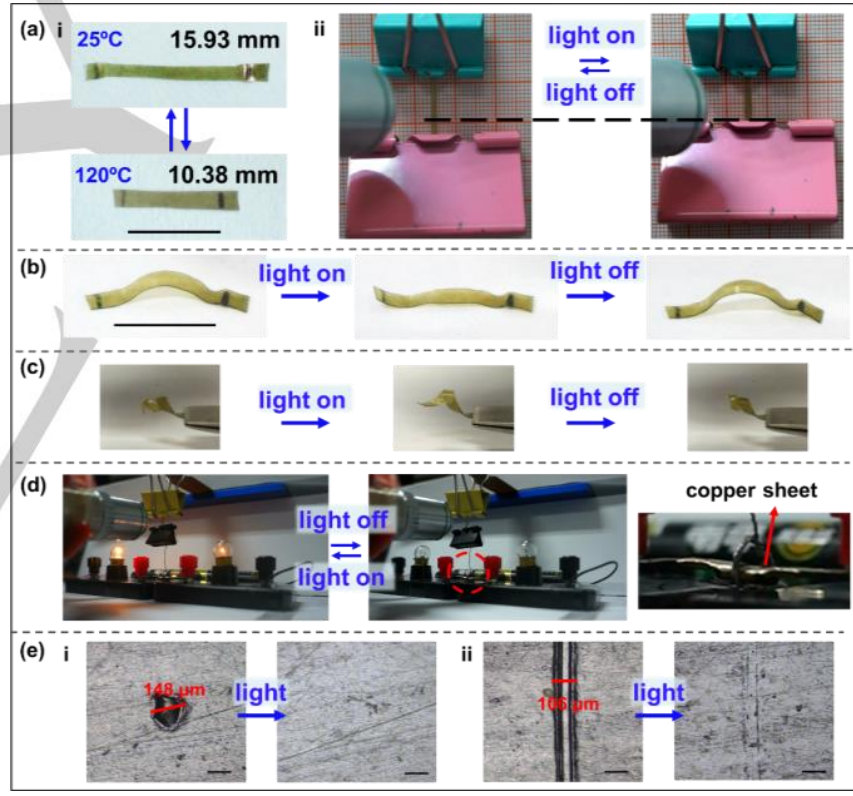

Figure 4. (a) Reversible actuation of the LCET-composite triggered by heat (i) and light (ii). The heavy of the pink binder clip is $8.8 \mathrm{~g}$, while the heavy of the sample is $4.2 \mathrm{mg}$. Infrared light intensity: $0.47 \mathrm{~W} / \mathrm{cm}^{2}$. Scale bar: $1 \mathrm{~cm}$. (b) Light triggered reversible actuation of a bending LCET-composite actuator. Infrared light intensity: $0.47 \mathrm{~W} / \mathrm{cm}^{2}$. Scale bar: $1 \mathrm{~cm}$. (c) Light triggered reversible actuation of a spiral LCET-composite actuator. Infrared light intensity: $0.47 \mathrm{~W} / \mathrm{cm}^{2}$. (d) Light triggered reversible stretching-contraction actuation controlling a circuit. Infrared light intensity: $0.47 \mathrm{~W} / \mathrm{cm}^{2}$. (e) Light triggered healing of the aligned LCET-composite with a needle pierced hole (i) and a cut (ii) by irradiating for $30 \mathrm{~min}$. Infrared light intensity: $0.84 \mathrm{~W} / \mathrm{cm}^{2}$. Scale bar: $100 \mu \mathrm{m}$ 


\section{Conclusion}

In summary, based on our recent finding and timetemperature equivalence principle, we here make it possible to reprocess traditional thermosets into 3D soft actuators by aligning and reconfiguring, which is considered impossible in the past. LCETs here not only have the good reprocessability like xLCEs, but also have incomparable stability which xLCEs do not have. Moreover, this quite stable alignment allows aligned LCETs to be locally and stepwise reprogrammed and welded to complex 3D soft actuators by heat, which is impossible by neither xLCE nor traditional thermosets previously, and which will enhance the complication of 3D shapes for different purposes and make more convenience in reprocessing. Besides traditional liquid crystalline epoxies, this idea may also be applicable to other traditional liquid crystalline thermosets with reversible and equilibrium reaction, such as polyurethanes (PU). In that case, it will dramatically promote the practical application of both traditional thermosets and 3D soft actuators in industry.

\section{Experimental Section}

Synthesis of the common epoxy (non-LC epoxy). We prepared the common epoxy by reacting diglycidyl ether of bisphenol A with adipic acid. Stoichiometric amount of diglycidyl ether of bisphenol A (1.00 $\mathrm{mmol}$ ) and adipic acid (1.00 mmol) (Figure S1) were mixed, and then heated to $180^{\circ} \mathrm{C}$ to melt and react. As the mixture became very viscous, it was cooled down to room temperature to afford a solid product which was not completely crosslinked. Then the mixture was sandwiched between two plates to be cured by a hot press for $6 \mathrm{~h}$ at $180^{\circ} \mathrm{C}$. A spacer was placed between the plates to control the thickness of films. The applied pressure was $6 \mathrm{MPa}$.

Synthesis of LCETs. We prepared LCETs by reacting diglycidyl ether of 4, 4'-dihydroxybiphenol (DGE-DHBP) with sebacic acid. Stoichiometric amount of DGE-DHBP $(1.00 \mathrm{mmol})$ and sebacic acid $(1.00 \mathrm{mmol})$ were mixed, and then heated to $180^{\circ} \mathrm{C}$ to melt and react. As the mixture became very viscous, it was cooled down to room temperature to afford a solid product which was not completely crosslinked. Then the mixture was sandwiched between two plates to be cured by a hot press for $6 \mathrm{~h}$ at $180^{\circ} \mathrm{C}$. A spacer was placed between the plates to control the thickness of films. The applied pressure was $6 \mathrm{MPa}$.

\section{Acknowledgements}

This research was supported by the National Natural Science Foundation of China (Nos. 21674057, 51722303, 21805159 and 21788102) and China Postdoctoral Science Foundation (Nos. 2018T110086 and 2017M620735).

Keywords: traditional liquid crystalline thermosets $\cdot 3 \mathrm{D}$ soft actuators $\cdot$ time-temperature equivalence principle $•$ reprogramming and welding $\cdot$ stable alignment
[1] a) D. Rus, M. T. Tolley, Nature 2015, 521, 467-475; b) R. V. Martinez, J. L. Branch, C. R. Fish, L. Jin, R. F. Shepherd, R. M. D. Nunes, Z. Suo, G. M. Whitesides, Adv. Mater. 2013, 25, 205-212; c) L. Hines, K. Petersen, G. Z. Lum, M. Sitti, Adv. Mater. 2017, 29, 1603483; d) L. Lu, W. Chen, Adv. Mater. 2010, 22, 3745-3748; e) M. Amjadi, K.-U. Kyung, I. Park, M. Sitti, Adv. Funct. Mater. 2016, 26, 1678-1698.

[2] A. D. McNaught, A. D. McNaught, Compendium of chemical terminology (IUPAC, the "Gold Book"), Blackwell Science, Oxford, 1997.

[3] a) C. N. Bowman, C. J. Kloxin, Angew. Chem. Int. Ed. 2012, 51, 4272 4274; b) C. J. Kloxin, C. N. Bowman, Chem. Soc. Rev. 2013, 42, 7161 7173; c) N. Zheng, Z. Fang, W. Zou, Q. Zhao, T. Xie, Angew. Chem. Int. Ed. 2016, 55, 11421-11425; d) Q. Zhao, W. Zou, Y. Luo, T. Xie, Sci. Adv. 2016, 2: e1501297; e) Y. Yang, Z. Pei, Z. Li, Y. Wei, Y. Ji, J. Am. Chem. Soc. 2016, 138, 2118-2121; f) Z. Pei, Y. Yang, Q. Chen, E. M. Terentjev, Y. Wei, Y. Ji, Nat. Mater. 2014, 13, 36-41; g) P. Taynton, K. Yu, R. K. Shoemaker, Y. Jin, H. J. Qi, W. Zhang, Adv. Mater. 2014, 26, 3938-3942; h) X. Qian, Q. Chen, Y. Yang, Y. Xu, Z. Li, Z. Wang, Y. Wu Y. Wei, Y. Ji, Adv. Mater. 2018, 1801103.

[4] a) Q. Chen, Y. Wei, Y. Ji, Chin. Chem. Lett. 2017, 28, 2139-2142; b) Q. Chen, Y. Yang, Y. Wei, Y. Ji, Acta Polym. Sin. 2019, 5, 451-468; c) W. Denissen, J. M. Winne, F. E. Du Prez, Chem. Sci. 2016, 7, 30-38; d) D. J. Fortman, J. P. Brutman, C. J. Cramer, M. A. Hillmyer, W. R. Dichtel, J. Am. Chem. Soc. 2015, 137, 14019-14022; e) D. Montarnal, M. Capelot, F. Tournilhac, L. Leibler, Science 2011, 334, 965-968.

[5] a) X. Sun, W. Wang, L. Qu, W. Guo, Y. Yu, H. Peng, Angew. Chem. Int. Ed. 2012, 51, 8520-8524; b) W. Wang, X. Sun, W. Wu, H. Peng, Y. Yu, Angew. Chem. Int. Ed. 2012, 51, 4644-4647; c) L. Wang, W. Liu, L. X. Guo, B.-P. Lin, X.-Q. Zhang, Y. Sun, H. Yang, Polym. Chem. 2017 8, 1364-1370; d) H. Yang, A. Buguin, J.-M. Taulemesse, K. Kaneko, S. Méry, A. Bergeret, P. Keller, J. Am. Chem. Soc. 2009, 131, 1500015004; e) C. Ohm, C. Serra, R. Zentel, Adv. Mater. 2009, 21, 48594862; f) Q. Chen, Y. Li, Y. Yang, Y. Xu, X. Qian, Y. Wei, Y. Ji, Chem. Sci. 2019, 10, 3025-3030; g) Y. Yang, X. Zhang, Y. Wei, Y. Ji, Acta Polym. Sin. 2017, 1662-1667; h) M. K. McBride, A. M. Martinez, L. Cox M. Alim, K. Childress, M. Beiswinger, M. Podgorski, B. T. Worrell, J. Killgore, C. N. Bowman, Sci. Adv. 2018, 4, eaat4634.

[6] a) M. Capelot, M. M. Unterlass, F. Tournilhac, L. Leibler, Acs Macro Lett. 2012, 1, 789-792; b) Y. Yang, S. Zhang, X. Zhang, L. Gao, Y. Wei, Y. Ji, Nat. Commum. 2019, 10, 3165.

[7] a) M. Giamberini, E. Amendola, C. Carfagna, Macromol. Chem. Phys. 1997, 198, 3185-3196; b) C. Carfagna, E. Amendola, M. Giamberini, Prog. Polym. Sci. 1997, 22, 1607-1647.

[8] Y. Yang, Y. Yang, S. Chen, Q. Lu, L. Song, Y. Wei, X. Wang, Nat. Commum. 2017, 8, 1559.

[9] a) M. Warner, E. M. Terentjev, Oxford Univ. Press, 2007; b) C. Ohm, M. Brehmer, R. Zentel, Adv. Mater. 2010, 22, 3366-3387; c) M. Yamada, M. Kondo, J. Mamiya, Y. Yu, M. Kinoshita, C. J. Barrett, T. Ikeda, Angew. Chem. Int. Ed. 2008, 47, 4986-4988; d) M. Camacho-Lopez, H. Finkelmann, P. Palffy-Muhoray, M. Shelley, Nat. Mater. 2004, 3, 307310; e) Y. Yu, M. Nakano, T. Ikeda, Nature 2003, 425, 145-145; f) A Buguin, M.-H. Li, P. Silberzan, B. Ladoux, P. Keller, J. Am. Chem. Soc. 2006, 128, 1088-1089; g) C. L. V. Oosten, C. W. Bastiaansen, D. J. Broer, Nat. Mater. 2009, 8, 677-682.

[10] F. I. Altuna, V. Pettarin, R. J. Williams, Green. Chem. 2013, 15, 33603366.

[11] a) F. Schwarzl, A. J. Staverman, J. Appl. Phys. 1952, 23, 838-843; b) S. Lyu, J. Schley, B. Loy, D. Lind, C. Hobot, R. Sparer, D. Untereker, Biomacromolecules 2007, 8, 2301-2310.

[12] J. S. Biggins, M. Warner, K. Bhattacharya, Phys. Rev. Lett. 2009, 103.

[13] S. Schuhladen, F. Preller, R. Rix, S. Petsch, R. Zentel, H. Zappe, Adv. Mater. 2014, 26, 7247-7251.

[14] H. Zeng, O. M. Wani, P. Wasylczyk, R. Kaczmarek, A. Priimagi, Adv. Mater. 2017, 29.

[15] S. Venkatraman, F. Boey, L. L. Lao, Prog. Polym. Sci. 2008, 33, 853874.

[16] A. Elias, F. Laurin, M. Kaminski, L. Gornet, Compos. Struct. 2017, 159, 228-239.

[17] K. Mizuno, J. Ishii, H. Kishida, Y. Hayamizu, S. Yasuda, D. N. Futaba M. Yumura, K. Hata, P. Natl. Acad. Sci. USA 2009, 106, 6044-6047.

[18] Y. Yang, Z. Pei, X. Zhang, L. Tao, Y. Wei, Y. Ji, Chem. Sci. 2014, 5, 3486-3492. 


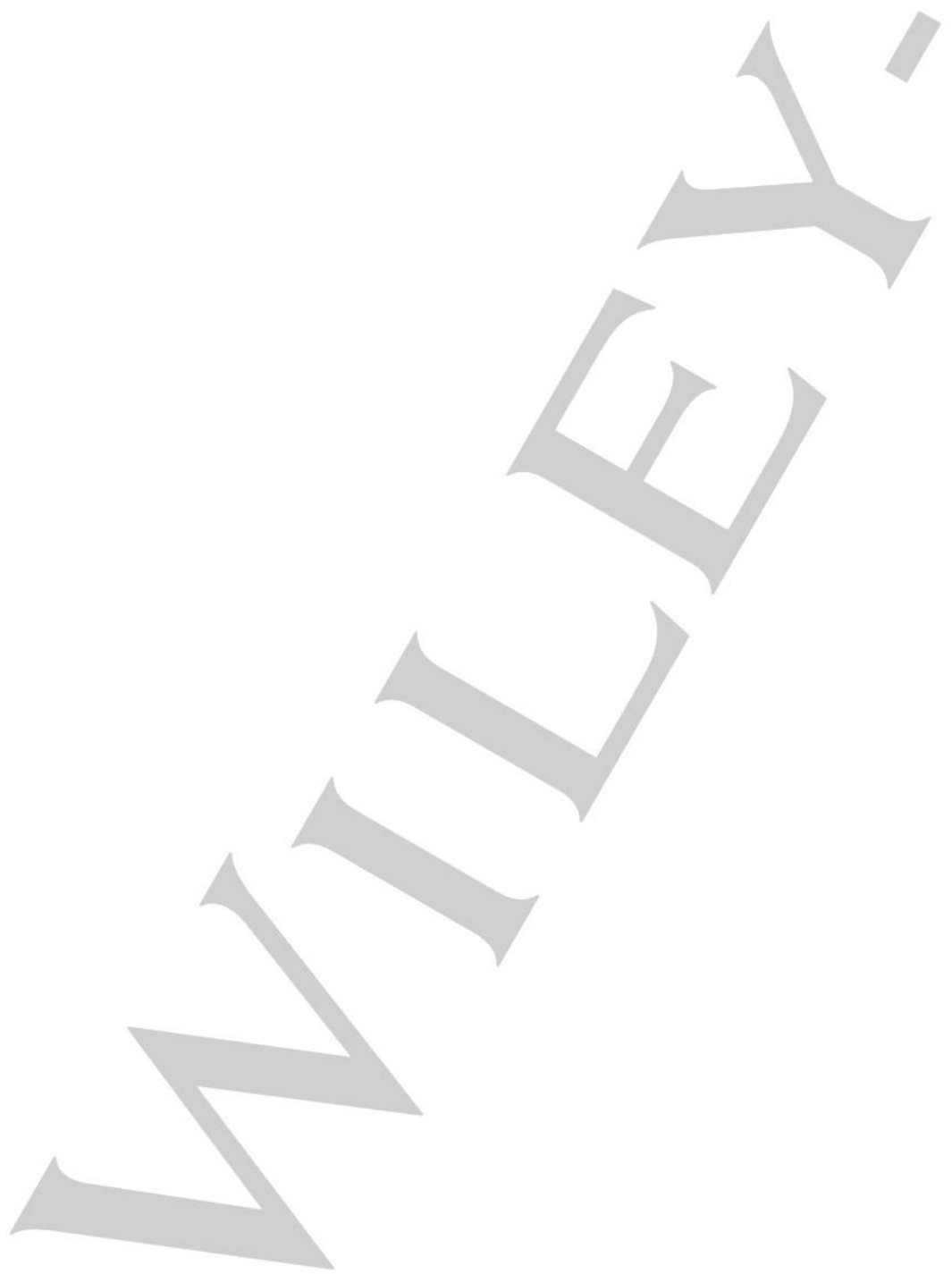

